\title{
INTESTINAL PHYTOBEZOAR
}

\author{
Levi Mark ${ }^{1}$
}

${ }^{1}$ Classified Specialist, Department of Surgery, INHS Sanjivani, Naval Base, Kochi, Kerala, India.

HOW TO CITE THIS ARTICLE: Mark L. Intestinal phytobezoar. J. Evolution Med. Dent. Sci. 2018;7(45):4929-4931, D0I: $10.14260 /$ jemds/2018/1097

\section{PRESENTATION OF CASE}

A 19-year-old naval cadet presented with abdominal pain of 03 hours duration. Pain was of sudden onset confined to the periumbilical region. Pain was colicky in nature without relieving or aggravating factors. There was no relevant past history. The individual was a vegetarian. On examination, the patient was afebrile, and vitals were stable. Abdominal examination revealed tenderness over the periumbilical region. Provisional diagnosis of Acute Appendicitis/ Intestinal Colic was made, and the patient was commenced on parenteral antibiotics and intravenous fluids. Thereafter, the patient had an episode of severe abdominal pain shifting to the left hypochondrium and lumbar region. There was no associated vomiting. Stool was present on rectal examination.

Blood and Urine values were grossly normal (TLC-7700, DLC: N71, L23, E02, M04: Amylase-34). NCCT Abdomen revealed- (i) Dilated centrally located small bowel loops, (ii) Large bowel displaced and compressed laterally, (iii) Rectum filled with air and faecal matter, (iv) Sudden transition of dilated small bowel loops in left lower abdomen with collapsed small bowel loops distal to the transition zone, (v) Minimal free fluid: no other acute signs. (Figure 1)

Phytobezoars occur in all age groups with absent physical findings. Small bowel Phytobezoars usually have an acute presentation with features of intestinal obstruction or perforation.(1) Radiological studies for detection of phytobezoars include Abdominal X-rays, Barium studies and USG and CT scans.(2) Currently, abdominal CT scans is the diagnostic modality of choice for detecting phytobezoars in the small bowel, as it can concurrently detects additional bezoars within the gastrointestinal tract. Surgery is the management of choice in small bowel phytobezoars. Definitive management consists of Exploratory Laparotomy with milking of contents into the caecum or performing an enterotomy with retrieval of contents.[3] During surgery it is mandatory to carry out a thorough exploration of the small intestine and colon to avoid recurrence of obstruction due to retained bezoar. Additionally, laparoscopic enterotomy is an effective less invasive treatment as it is associated with shorter hospital stay, early return of GI function and recovery time.(3)

Gastrointestinal bezoar obstruction is uncommon and accounts for only 0.4 - $4 \%$ of all intestinal obstruction cases. In this paper, we present a rare case of intestinal phytobezoar.

'Financial or Other Competing Interest': None.

Submission 24-09-2018, Peer Review 19-10-2018,

Acceptance 25-10-2018, Published 05-11-2018.

Corresponding Author:

Dr. Levi Mark,

Classified Specialist,

Department of Surgery,

INHS, Patanjali,

Naval Base,

Karwar-581308, Karnataka, India.

E-mail: drlmark@rediffmail.com

DOI: $10.14260 /$ jemds $/ 2018 / 1097$

\section{(c) (1) $(9)$}

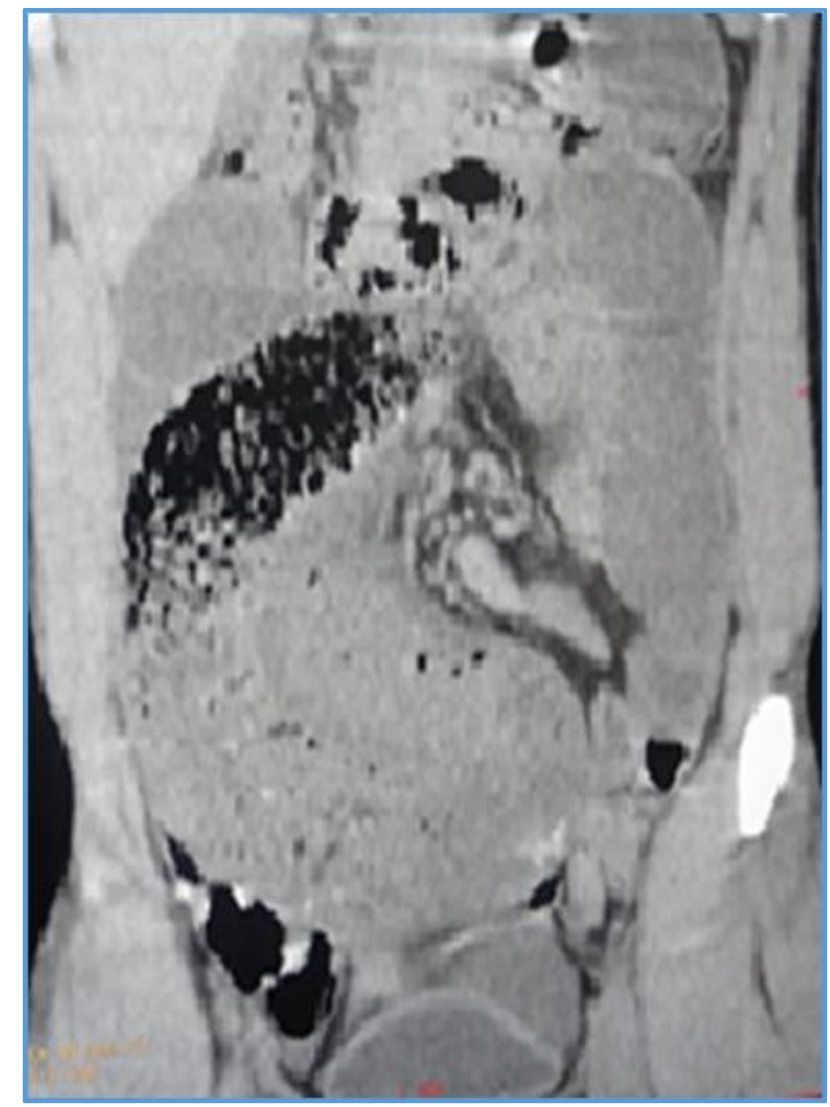

Figure 1. Coronal CECT Image shows Characteristic Small Bowel Faeces Sign (Arrow), Air Density within the Bowel consisting of Gas and Digested Particulate Proximal to Site of Obstruction

\section{DIFFERENTIAL DIAGNOSIS}

A provisional diagnosis of Acute Small Bowel Obstruction was made due to? Bands. Ryle's tube was inserted with an attempt to ease the obstruction as part of conservative management. However, the next day, the patient continued to have pain with an associated episode of vomiting. He was taken up for Exploratory Laparotomy in view of non-improvement with conservative management.

\section{CLINICAL DIAGNOSIS}

Intraoperative findings revealed- (1) Serous fluid in the peritoneal cavity, (2) Dilated proximal ileal loops and collapsed caecum, (3) A coagulum of vegetable matter containing guava seeds in a fist-sized indentable mass occluding the proximal lumen and (4) No bands, strictures, growths or enlarged lymph nodes were present. As it was not possible to milk the mass into the caecum/ ascending colon, an enterotomy was made over the ileum and the coagulum of guava seeds pulp was evacuated. The enterotomy was closed and the patient made an uneventful recovery and was discharged. The final diagnosis was phytobezoar causing acute intestinal obstruction following consumption of guavas. 


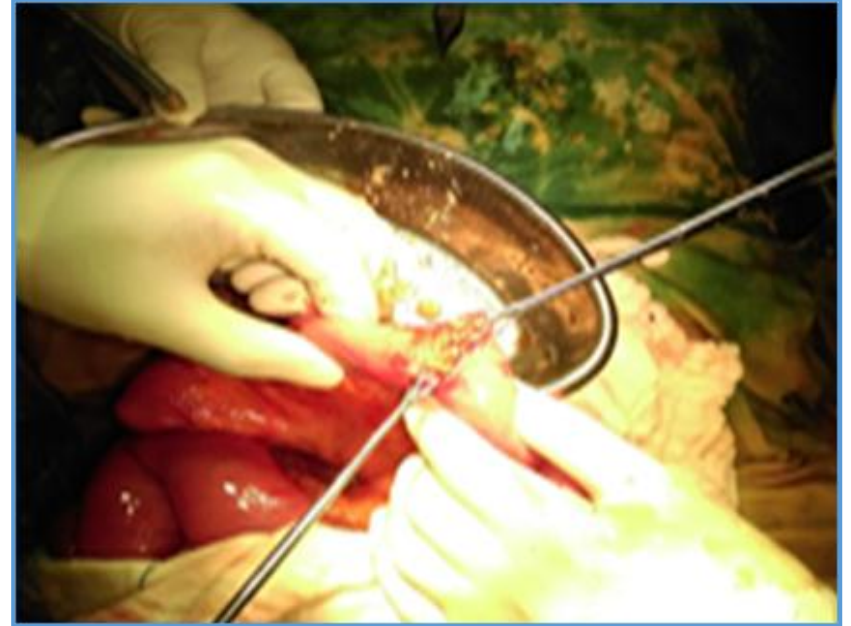

Figure 2. Image shows Enterotomy done and Multiple Guava Seeds being Extracted Out

\section{PATHOLOGICAL DISCUSSION}

The word bezoar is derived from either the Arabic word (Badzehr) or the Persian word (Padzehr), both of which denote counter poison or antidote. This word applied to a greenish, hard concretion found in the fourth stomach of the Syrian Goat. [4] Bezoar is an agglomeration of food or foreign material in the intestinal tract; ingested foreign material that accumulates within the GI tract. Bezoar is classified according to the primary constituent as Trichobezoar (Hair), Phytobezoar (Plant material), Pharmacobezoar (Medicine) but may fall into miscellaneous category including fungal agglomerations, food boluses, chemical concretions or foreign bodies.[5] Although, phytobezoars predominate most bezoars are seen in the stomach, but occur anywhere from the oesophagus to the rectum.

Bezoars have been reported in all age groups. The pathogenesis of bezoar remains unclear and appears to be multifactorial in the adult population. It appears that alteration in gastric anatomy and physiology as well as overindulgence in high fibre diets, abnormal mastication, psychiatric disorders play an important role.[6] Phytobezoars are composed of vegetable matter indigestible by man and contain cellulose, hemi-cellulose, lignin and fruit tannin's.[4] The greater propensity for phytobezoars to cause obstruction is thought to be due to the fact that they are often multiple, of harder consistency and more irregular in surface. The presence of bezoars should always be considered in individuals with recurring nausea, vomiting and epigastric pain or those who have small bowel obstruction without any previous history of antecedent intra-abdominal surgery. A careful and detailed history may suggest the diagnosis preoperatively.[7]

Primary small bowel Bezoars are rare and almost always obstructive. Phytobezoars can develop secondarily in individuals with underlying small bowel disease (Diverticulitis, Crohn's disease, TB, Strictures and Tumours)(1). Impaired mastication may also contribute to bezoar formation in older patients. Various factors may be involved in bezoar formation that include changes in the production of acid, pepsin and mucus as well as abnormality in the grinding mechanism and interdigestive migratory motor complex.(5,6)
In the normal stomach, vegetable fibres which cannot pass through the pylorus undergo hydrolysis within softening them enough to pass through the small bowel. Phytobezoars are concretions composed of vegetable matter such as skin, seeds and fibres of fruits and vegetables that are poorly digested and found in the alimentary tract.(4) Predisposing factors to bezoar formation are sequelae of gastric surgery: Gastric resection, partial gastrectomy or truncal vagotomy with pyloroplasty,(6) ingestion of high fibre foods, abnormal mastication, diminished gastric secretions and motility, autonomic neuropathy in diabetes mellitus and myotonic dystrophy. $(8,1)$

Bezoars usually form in the stomach and can pass into the small bowel where they occasionally cause obstruction. Most bezoars are found in patients with a history of previous gastric surgery. Abdominal pain and vomiting are the common symptoms associated with bezoars and small bowel dilatation, leukocytosis are the frequent presenting signs.

Phytobezoars usually become impacted in the narrowest portion of the small bowel, the commonest site being the terminal ileum followed by the jejunum. ${ }^{[4]}$ Radiography includes X-ray abdomen which shows a dilated obstructive pattern and occasionally the outline of a bezoar may be visualised. Barium studies show an intraluminal filling defect of variable size that is not fixed to the bowel wall with barium filling the interstices. Ultrasound along with CT abdomen can provide an accurate diagnosis with the possibility of exploring various treatment options. USG findings for phytobezoars include arc-like surfaced intraluminal mass, a strong posterior acoustic shadow of the mass and a twinkling artifact in front of the mass. CT imaging may vary from a round, nonhomogeneous mottled gas patterned intraluminal mass just proximal to the transitional zone to a soft tissue mass without gas.[2] However, the diagnostic value of CT abdomen needs to be emphasised, as it can exclude other causes of acute abdomen becoming the first line of examination.(1)

Previous gastric resection or ulcer surgery such as partial gastrectomy or truncal vagotomy with pyloroplasty predisposes to bezoar formation. Other predisposing factors are ingestion of high fibre foods, abnormal mastication, diminished gastric secretions and motility, autonomic neuropathy and myotonic dystrophy. Phytobezoars can also develop secondarily if there are areas of sufficient stagnation within a dilated bowel segment as may occur in patients with strictures caused by Crohn's disease, TB, h/o previous surgery or in small bowel diverticulae. ${ }^{[4]}$

\section{DISCUSSION OF MANAGEMENT}

Management of intestinal phytobezoars is primarily by operative management. Exploration is done either by laparoscopic or open method to relieve the obstruction. Operative interventions include milking of the bezoar into the large bowel without enterotomy after compressing and fragmenting it intraluminally, thereby maintaining the small bowel integrity and avoiding peritoneal spillage and contamination.[7],[9] Enterotomy and bezoar extraction is only reserved for those cases as a last resort where fragmentation and milking of the bezoar is not possible.[9]

\section{FINAL DIAGNOSIS}

Phytobezoar causing acute intestinal obstruction following consumption of guavas. 


\section{REFERENCES}

[1] Teng HC, Nawawi O, Ng KL, et al. Phytobezoar: an unusual cause of intestinal obstruction. Biomed Imaging Interv J 2005;1(1):e4.

[2] Lee $\mathrm{KH}, \mathrm{Han} \mathrm{HY}$, Kim $\mathrm{HJ}$, et al. Ultrasonographic differentiation of bezoar from feces in small bowel obstruction. Ultrasonography 2015;34(3):211-6.

[3] Das SS, Hussain M, Bhat A, et al. Phytobezoar causing terminal ileal obstruction - Can enterotomy be avoided? JSM Clin Case 2014;2(2):1024.

[4] Chisholm EM, Leong HT, Chung SC, et al. Phytobezoar: an uncommon cause of small bowel obstruction. Annals of the Royal College of Surgeons of England 1992;74(5):342-4.

[5] Pankaj S, Kumar S, Kumar B, et al. Rapunzel syndrome: a rare case of vomiting and pain abdomen. JK Science 2012;14(3):146-8.
[6] Agaba EA, Ikedilo O, Vemulapalli P. Small intestinal bezoar after gastric bypass: an unusual cause of acute intestinal obstruction at the Jejunojejunal anastomosis. Journal of Obesity and Bariatrics 2015;2(1):1-4.

[7] Singh N, Digray NC, Parihar S, et al. Phytobezoar induced ileal obstruction in children - report of two cases. JK Science 2006;8(2):103-5.

[8] Huang LC, Lin HH, Huang SC, et al. Gastrointestinal bezoar: clinical study of 15 years' experience from a medical center in Eastern Taiwan. Tzu Chi Med J 2005;17(2):83-6.

[9] Krausz MM, Moriel EZ, Ayalon A, et al. Surgical aspects of gastrointestinal persimmon phytobezoar treatment. Am J Surg 1986;152(5):526-30. 